\title{
Prescription drug abuse rising among Aboriginal youths
}

I ndications of a staggering level of prescription drug abuse by Aboriginal youths have left experts urging a more coordinated approach to the problem.

Some $18.4 \%$ of Inuit youths aged $12-17,11 \%$ of Aboriginal youths and $8.8 \%$ of Metis youths living in urban Canada - as compared with $5.6 \%$ of non-Aboriginal youths — self-report abuse of prescription drugs including sedatives, stimulants and pain relievers, according to Cheryl Currie, an epidemiologist and an assistant professor of public health in the Faculty of Health Sciences at the University of Lethbridge in Alberta.

Responses to Health Canada's national Youth Smoking Survey indicated that abuse of prescription painkillers was five times greater among Inuit youth than among nonAboriginal youth, Currie said in a presentation of her findings at the Canadian Public Health Association's annual conference in Edmonton, Alberta (http://resources.cpha.ca /CPHA/Conf/Data/2012/A12-086e.pdf).

The migration of many Inuit people to southern Canada has contributed to prescription drug abuse as youths in urban centres are exposed to greater degrees of racism and discrimination, Currie surmised. "Inuit people are urbanizing and within that population there are high rates of drug abuse. And there are also high rates of social exclusion as evidenced by this and other studies. It could be a contributing factor to drug abuse."

Currie adds that the survey indicated a correlation between prescription drug abuse and feelings of exclusion at school. "I would like to see school policies that recognize that unequal treatment may be driving substance abuse in First Nations, Metis and Inuit children as a way of coping with adversity."

Others, though, caution that the Currie's conclusions are based on limited evidence. The sample size of the study is small, notes Russell Callaghan, a

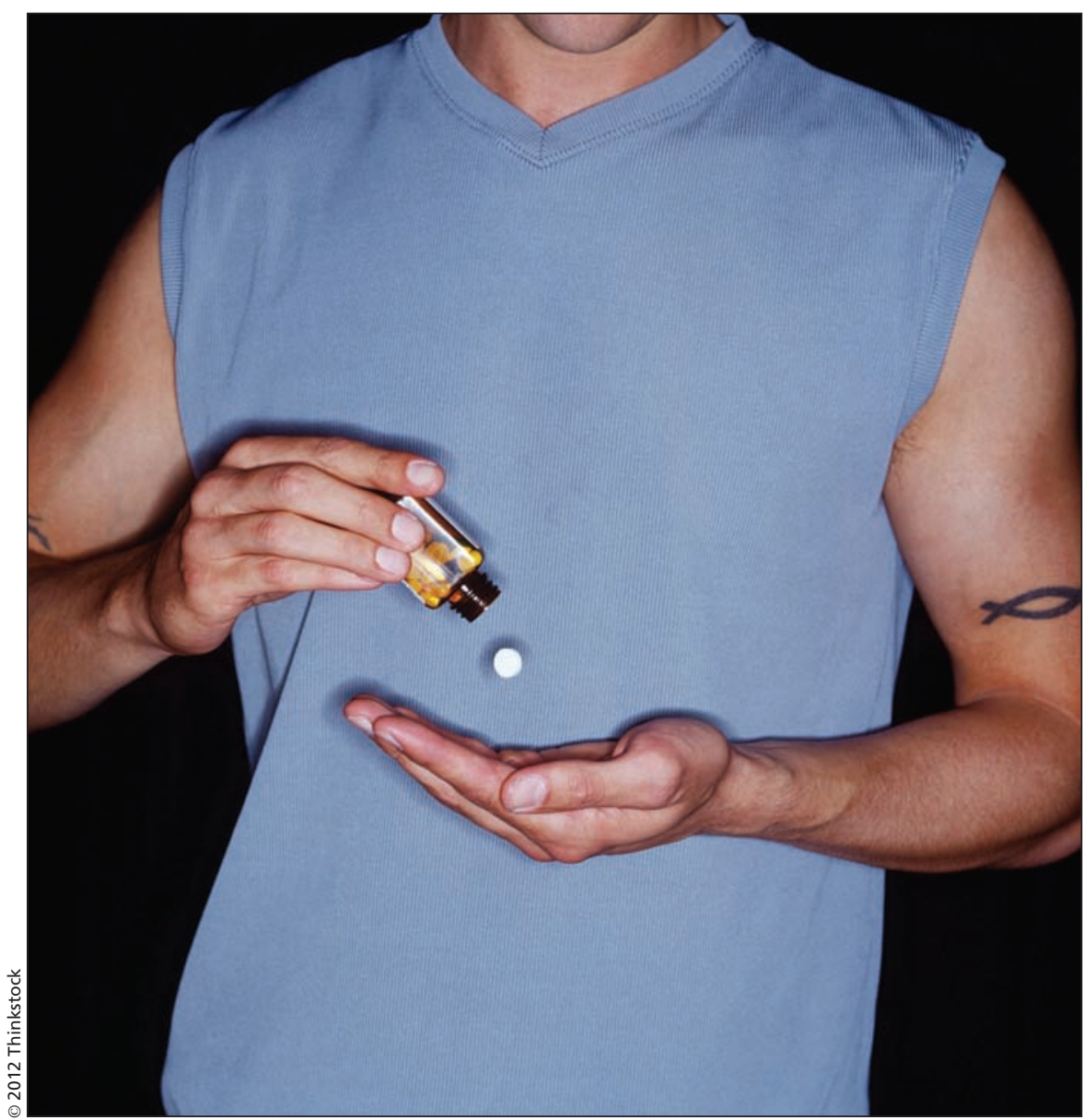

A national survey indicated that abuse of prescription painkillers was five times greater among Inuit youth than among non-Aboriginal youth.

researcher at the Centre for Addictions and Mental Health in Toronto, Ontario.

Other gauges of prescription drug abuse among Aboriginal youths are sparse. The recently released First Nations Regional Health Survey 2008/10 indicated that the use of sedatives/sleeping pills among First Nations youth almost tripled from $0.8 \%$ in 2003 to $2.2 \%$ in 2010 but the survey did not track the use of prescription opioids (www.rhs-ers.ca/sites/default/files/RHS _Phase_II_-_Key_Findings.pdf).

With respect to Inuit youth, the data on prescription drug misuse lacks coherence because "there is no system in place to track usage," says Gwen Watts, manager of health education programs for the Nunatsiavut govern- ment in Happy Valley-Goose Bay. But "you can get pretty much whatever [illicit drugs] you want in our communities," adds the former manager of mental health and addiction programs for Nunatsiavut.

According a recent review of substance use-related services and supports for First Nations people, the lack of accurate surveillance data makes it difficult to assess the extent of prescription drug abuse, "particularly as it relates to illicit supply sources (thefts, organized crime, family members procuring or using another family member's prescription, and internet supply)."

The review of the efficacy of the federal government's National Native Alcohol and Drug Abuse Program 
(NNADAP), which operates a network of on-reserve addiction services including 58 centres and more than 550 community-based prevention programs, also resulted in the creation of a "national framework" to address substance abuse among Aboriginal peoples (http://nnadaprenewal.ca/wp -content/uploads/2012/01/Honouring-Our -Strengths-2011_Eng1.pdf).

A "comprehensive approach" is needed to resolve substance abuse problems, argues Honouring Our Strengths: A Renewed Framework to Address Substance Use Issues Among First Nations People in Canada, which was developed by the National Native Addictions Partnership Foundation, the Assembly of First Nations and Health Canada.

Community-based pilot projects undertaken by Health Canada in recent years indicate that "certain key elements are important for successful interventions at the community level; the development of steering or advisory committees to guide prevention efforts at a community/regional level; support from chiefs in council; collaboration with police, schools, family services and health centres; community consultations; use of the mass media; multidisciplinary team approaches; and showing people how much of a problem it is, using local data," the framework document states.

It also urges more intensive substance abuse services for those addicted to prescription drugs, and specialized treatment, including "medically-based detoxification and psychiatric services, as well as culturallybased interventions" for those with concomitant mental health disorders or other conditions such as Fetal Alcohol Spectrum Disorder.

As well, the framework urges a host of other measures, including:

- "A multi-faceted approach to address the issues of prescription drug abuse, including exploration of traditional medicine and alternative therapies; improving access to counselling services; reducing peer pressure; and putting in place education and prevention strategies aimed at youth, doctors, pharmacists, dentists, police and the general public.

- Infrastructure to support collaboration among the First Nations and Inuit Health Branch staff, NNADAP staff, doctors, pharmacists, police and band councils to lessen 'double doctoring.' This includes raising awareness of First Nations health issues within bodies such as the Royal College of Physicians and Surgeons of Canada, the College of Family Physicians, the Canadian Medical Association, and medical schools. It may also include development of an early warning system for prescription drugs use/abuse in First Nations communities.

- Cooperative approaches with physicians and pharmacists to support appropriate prescribing at the community level."

Resolving the problem will require greater awareness among prescribers, argues Carol Hopkins, executive director of the National Native Addictions Foundation, based in Muskoday, Saskatchewan. "They need to screen for the likelihood of addiction in a way that is culturally sensitive."
Thus far, the framework has engendered a favourable response. "This was developed with honest collaborative community input that brings together western and indigenous understandings," says Colleen Dell, research chair in substance abuse in the School of Public Health at the University of Saskatchewan in Saskatoon. "This document underscores the importance of culture and community in health well-being and the importance of research in documenting what really works in communities. We strongly hope the government will continue to support the process and that this framework will be implemented by the government and the communities."

The federal government has also asked the Canadian Centre on Substance Abuse to coordinate national efforts to tackle prescription drug misuse in Aboriginal communities.

In the past, the federal response was marred by "false starts," says Deborah Cumming, national priority adviser for the centre. While it was recognized that Aboriginal communities are a "hot spot" that require special attention, "nobody wanted to deal with this."

To date, the effort to coordinate a response has been entirely organizational, Cumming says, adding that federal action will fall under the authority of Health Canada's Prescription Drug Abuse Coordinating Committee, which recently was expanded to include the Assembly of First Nations, the Canadian Centre on Substance Abuse, and the National Native Addictions Partnership Foundation. - Paul Christopher Webster, Toronto, Ont.

CMAJ 2012. DOI:10.1503/cmaj.109-4258 